\title{
Remote Individual Addressing of Quantum Emitters with Chirped Pulses
}

\author{
S. Casulleras $\odot,{ }^{1,2,}$ C. Gonzalez-Ballestero $\odot,{ }^{1,2}$ P. Maurer $\odot,{ }^{1,2}$ J. J. García-Ripoll@ ${ }^{3}$ and O. Romero-Isart ${ }^{1,2}$ \\ ${ }^{1}$ Institute for Quantum Optics and Quantum Information of the Austrian Academy of Sciences, 6020 Innsbruck, Austria \\ ${ }^{2}$ Institute for Theoretical Physics, University of Innsbruck, 6020 Innsbruck, Austria \\ ${ }^{3}$ Instituto de Física Fundamental IFF-CSIC, Calle Serrano 113b 28006 Madrid, Spain
}

(Received 20 May 2020; accepted 29 January 2021; published 9 March 2021)

\begin{abstract}
We propose to use chirped pulses propagating near a band gap to remotely address quantum emitters. We introduce a particular family of chirped pulses that dynamically self-compress to subwavelength spot sizes during their evolution in a medium with a quadratic dispersion relation. We analytically describe how the compression distance and width of the pulse can be tuned through its initial parameters. We show that the interaction of such pulses with a quantum emitter is highly sensitive to its position due to effective Landau-Zener processes induced by the pulse chirping. Our results propose pulse engineering as a powerful control and probing tool in the field of quantum emitters coupled to structured reservoirs.
\end{abstract}

DOI: 10.1103/PhysRevLett.126.103602

An exciting platform in quantum optics, both in the microwave [1-8] and the optical [9-19] regime, is obtained by coupling quantum emitters to photonic structures where band gaps and dispersion relations can be engineered. In essence, these systems allow enhancing and tailoring subwavelength light-matter interaction and bath-mediated coupling between quantum emitters. There are multiple applications in the context of quantum simulation $[12,13]$ and computation $[4,7,15]$, as well as in exploring unconventional quantum optics $[1-3,5,6,8-11,14,16-19]$. Most of these setups rely on, or would benefit from, the possibility of electromagnetically addressing individual quantum emitters. However, such addressing can be challenging due to, for instance, insufficient (e.g., subwavelength) separation between contiguous emitters or phase mismatch between outside radiation and the electromagnetic modes of the structure. Even in platforms where local probes are available, such as superconducting circuits, these probes might introduce unwanted decoherence and lack the flexibility that a fully tunable local probe could provide. A potential route toward such individual addressing could be paved by active electromagnetic engineering, where not only the dispersion relation but also the time-dependent state of the electromagnetic environment is tailored.

In this Letter, we explore the possibility of exploiting active engineering in structured electromagnetic reservoirs. In particular, we introduce a specific family of chirped electromagnetic pulses and show that, in a medium displaying a quadratic dispersion relation above a band gap, their free evolution causes them to dynamically self-compress into a single, potentially subwavelength spot. Self-compression of chirped pulses using materials with nonlinear electromagnetic response (e.g., with intrinsic Kerr nonlinearities) have been exploited before [20-22]. In contrast, here we use nonlinear dispersion relations that can be engineered with linear lossless materials (e.g., photonic crystals). We then study the interaction between these chirped pulses and a quantum emitter, demonstrating the strong impact of the pulse self-compression on the dynamics of the emitter. Specifically, a quantum emitter situated at the compression spot is shown to display radically different dynamics than a quantum emitter situated at any other position. Our results therefore suggest that chirped pulses in structured electromagnetic media can be used to remotely address individual quantum emitters within an array with subwavelength separation [see Fig. 1(a) for a schematic representation]. While we discuss our results in the context of structured photonic reservoirs, our results can be extended to other implementations where bosonic excitations propagating near a band gap couple to quantum emitters (e.g., phononic networks coupled to color centers in diamond [23]).

More specifically, we consider an electromagnetic medium extended along the $z$ axis displaying an energy band gap for electromagnetic modes propagating along $z$ with wave vector $\mathbf{k}=k \mathbf{e}_{z}$. The band gap is characterized by a cutoff frequency $\omega_{c}$, below which there are no $z$-propagating modes. We consider that for frequencies $7 \omega \gtrsim \omega_{c}$ the dispersion relation of the propagating modes can be written as

$$
\omega(k)=\omega_{c}+\frac{v^{2}}{2 \omega_{c}} k^{2} .
$$

Here $v$ is a dimensional parameter characterizing the band curvature. We assume the $z$-propagating electromagnetic modes to be tightly confined in the transverse $(x, y)$ plane in order to increase the interaction with quantum emitters, as discussed later. The propagating electromagnetic modes for a given polarization can then be described by a single mode index, namely, their longitudinal wave number $k$, and the single band Eq. (1). As mentioned before, the considered 


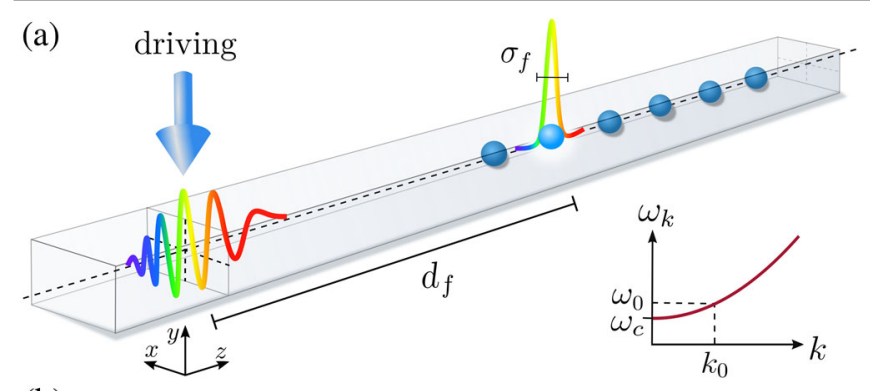

(b)
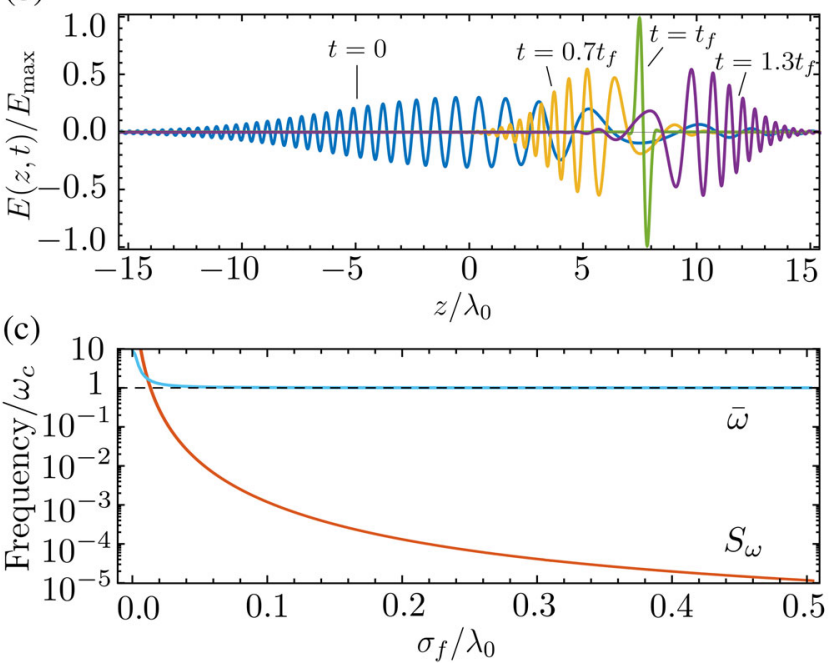

FIG. 1. (a) Quantum emitters embedded in an electromagnetic waveguide. A time-dependent driving applied at the origin of the coordinate system creates a chirped self-compressing electromagnetic pulse. At a time $t_{f}$ the pulse becomes compressed at a distance $d_{f}$ from the origin, reaching a minimum width $\sigma_{f}$. Inset: Quadratic dispersion relation of the waveguide. The distribution of the pulse wave number along $z$ is centered around $k_{0}=2 \pi / \lambda_{0}$. (b) Spatial profile of the electric field of the chirped pulse at different times. The electric field is normalized by its maximum value $E_{\max }=\max _{d, t} E(d, t)$. (c) Mean frequency $\bar{\omega}$ and standard deviation $S_{\omega}$ (defined in the text) of the electric field pulse as a function of the compression width $\sigma_{f}$. Parameters used: $\omega_{0} / \omega_{c}=1.005, d_{f} / \lambda_{0}=7.5, \sigma_{f} / \lambda_{0}=0.21, \phi=0$.

electromagnetic medium can be implemented either in the microwave regime or in the optical regime.

In the medium defined above, we focus on the time dynamics of a single component of the electric field as a function of $z$ evaluated at a given position in the transverse plane, say $\left(x_{0}, y_{0}\right)$. We label such scalar electric field as $E(z, t)=2 \operatorname{Re}\left\{E^{+}(z, t)\right\}$. As discussed later, $E(z, t)$ is relevant to describe the electric-dipole interaction with a quantum emitter placed at $\left(x_{0}, y_{0}, z\right)$. The first main result of this Letter is to propose and parametrize a particular family of chirped electromagnetic pulses that dynamically self-compress due to the dispersion relation given by Eq. (1). These pulses depend on five real parameters $\left(k_{0}, d_{f}, \sigma_{f}, \phi, N\right)$, defined below, and can be written as

$$
E^{+}(z, t) \equiv\left|E^{+}(z, t)\right| e^{i \theta(z, t)} e^{i \phi} e^{i\left(k_{0} z-\omega_{0} t\right)} .
$$

Here $k_{0}$ is the carrier wave number with corresponding frequency $\omega_{0} \equiv \omega\left(k_{0}\right)$ and $\phi$ is a constant phase. The amplitude of the pulse is given by

$$
\left|E^{+}(z, t)\right| \equiv \frac{N}{k_{c} \sigma(t)} \exp \left[-\frac{\sigma_{f}^{2}}{2 \sigma^{4}(t)}\left(z-\frac{v t}{\eta}\right)^{2}\right],
$$

where $k_{c} \equiv \omega_{c} / v$ and $\eta \equiv k_{c} / k_{0}$. The time-dependent pulse width is given by

$$
\sigma(t) \equiv \sqrt[4]{\sigma_{f}^{4}+\frac{s^{2}\left(d_{f}, t\right)}{k_{c}^{4}}},
$$

where $s(z, t) \equiv \eta k_{c} z-\omega_{c} t$ is a spatiotemporal dimensionless function, $\sigma_{f}$ is the spot size, and $d_{f}$ is the focal distance. The constant $N$ is a pulse amplitude parameter. The time-dependent phase in Eq. (2), which is responsible for the chirping, is given by

$$
\theta(z, t) \equiv-\frac{s\left(d_{f}, t\right) s^{2}(z, t)}{2 \eta^{2} k_{c}^{4} \sigma^{4}(t)}+\frac{1}{2} \arctan \left[\frac{s\left(d_{f}, t\right)}{k_{c}^{2} \sigma_{f}^{2}}\right] .
$$

The pulse $E(z, t)$ is shown in Fig. 1(b) at four particular instants of time, taking $k_{0}>0$ (it propagates rightward). At $t=0$ the pulse, centered at $z=0$, is down-chirped, i.e., the wavelength at the front of the pulse is larger than at its tail. As time increases, free evolution in the quadratic dispersion relation induces self-compression of the pulse. Specifically, the width $\sigma(t)$ in Eq. (4) becomes smaller following the decrease of the function $s\left(d_{f}, t\right)$. Maximum compression occurs at a specific time $t_{f} \equiv \eta d_{f} / v$, where the width reaches its minimum $\sigma\left(t_{f}\right)=\sigma_{f}$ and the spatial extension of the pulse is minimized around a compression point $z=d_{f}$. At this time, all the components of the pulse sync in phase, namely, $\theta\left(z, t_{f}\right)=0$. At latter times $t>t_{f}$ the pulse becomes up-chirped and it expands in size. In principle, the compression distance $d_{f}$ and width $\sigma_{f}$ of the pulse can be arbitrarily chosen, allowing for deep subwavelength compression $\left(\sigma_{f} \ll \lambda_{0} \equiv 2 \pi / k_{0}\right)$. In Fig. 1(c), we show the mean frequency $\bar{\omega} \equiv \int_{\mathbb{R}} \omega p(\omega) d \omega$ and standard deviation $S_{\omega} \equiv\left[\int_{\mathbb{R}}(\omega-\bar{\omega})^{2} p(\omega) d \omega\right]^{1 / 2}$ of the pulse at $z=0$ as a function of the compression width $\sigma_{f}$, using $p(\omega) \equiv$ $|\tilde{E}(0, \omega)| / \int_{\mathbb{R}}|\tilde{E}(0, \omega)| d \omega$ with $\tilde{E}(z, \omega) \equiv(2 \pi)^{-1 / 2} \int_{\mathbb{R}} E(z, t)$ $\exp (-i \omega t) d t$. Stronger compression (lower $\sigma_{f}$ ) requires higher mean pulse frequencies and wider distributions in frequency space. We consider hereafter sufficiently large spot sizes and small carrier wave numbers, say $\sigma_{f} \gtrsim 10^{-1} \lambda_{0}$ and $k_{0} \lesssim 10^{-1} \omega_{c} / v$, such that the spectral properties of the pulse are consistent with the assumptions considered (e.g., single quadratic band approximation). The frequency distribution of the pulse does not significantly depend on $d_{f}$. 
One can show that $E(z, t)$, as defined above, is consistent within electrodynamics in the medium Eq. (1). Indeed, $E(z, t)$ has been constructed as a particular linear combination of electromagnetic field modes, engineered in analogy to the wave packet contracting quantum dynamics of a massive particle evolving in free space, which also displays a quadratic dispersion relation (see the Supplemental Material of [24]). The chirped electromagnetic pulses can be produced by driving the waveguide at a given spatial position, say at $z=0$. In the Supplemental Material of this Letter [25], we provide two detailed examples of how the chirped pulses $E(z, t)$ can be engineered in a 3D hollow waveguide with perfectly conducting walls [26], a relevant system for circuit quantum electrodynamics [31,32], and in a multilayer photonic crystal.

Let us now address the interaction between the selfcompressing chirped pulse $E(z, t)$ and a single quantum emitter placed at the position $\left(x_{0}, y_{0}, d\right)$. The quantum emitter is first modeled as a qubit with electronic levels $\{|g\rangle,|e\rangle\}$ and transition frequency $\omega_{q}$. Its electric-dipole moment is assumed to point along the direction of the component of the electric field considered in $E(z, t)$. Accordingly, the Hamiltonian describing the electric-dipole interaction of the qubit with the electromagnetic pulse is given by

$$
\frac{\hat{H}}{\hbar}=\frac{\omega_{q}}{2} \hat{\sigma}_{z}+\frac{\Omega(d, t)}{2} \hat{\sigma}_{+}+\frac{\Omega^{*}(d, t)}{2} \hat{\sigma}_{-},
$$

where $\Omega(d, t) \equiv-2 d_{e g} E(d, t) / \hbar$ is the time- and positiondependent Rabi coupling strength, $d_{e g}$ is the dipole matrix element of the qubit, and $\hbar$ is the reduced Planck constant. We use the Pauli matrix operators for the qubit levels $\quad \hat{\sigma}_{z} \equiv|e\rangle\langle e|-| g\rangle\langle g| \quad$ and $\quad \hat{\sigma}^{+} \equiv\left[\hat{\sigma}^{-}\right]^{\dagger}=|e\rangle\langle g|$. The dynamics of the state of the qubit $\hat{\rho}(t)$ including spontaneous emission with rate $\Gamma$ are modeled with the Born-Markov master equation $\partial_{t} \hat{\rho}=(i \hbar)^{-1}[\hat{H}, \hat{\rho}]+$ $\Gamma\left(\hat{\sigma}_{-} \hat{\rho} \hat{\sigma}_{+}-\left[\hat{\sigma}_{+} \hat{\sigma}_{-}, \hat{\rho}\right]_{+} / 2\right)$, which can be numerically solved. We remark that the rotating wave approximation, namely, using $\Omega(d, t) \equiv-2 d_{e g} E^{+}(d, t) / \hbar$ in Eq. (6), can be employed in the regime $\Omega_{0} \ll 2 \omega_{q}$ where $\Omega_{0} \equiv \max _{d, t}|\Omega(d, t)|$.

Let us analyze the dynamics of a qubit situated at position $z=d$ and which is initially in the ground state $\hat{\rho}(0)=|g\rangle\langle g|$. Figure 2(a) shows the excited state probability $p_{e}(d, t)=\operatorname{tr}[\hat{\rho}(t)|e\rangle\langle e|]$ as a function of time for different positions $d$ of the qubit. When the qubit is situated at the compression distance $\left(d=d_{f}\right)$, the qubit is excited when the pulse reaches it at $t=t_{f}$ and deexcited when it travels further away. Hence, $p_{e}\left(d_{f}, t \gg t_{f}\right) \approx 0$. However, when the qubit is situated far from the compression distance $\left(\left|d-d_{f}\right| \gg \sigma_{f}\right)$, it remains excited at long times $p_{e}\left(d \neq d_{f}, t \gg t_{f}\right) \approx 1$. The interaction of the qubit with the pulse happens at a timescale shorter than $\Gamma^{-1}$

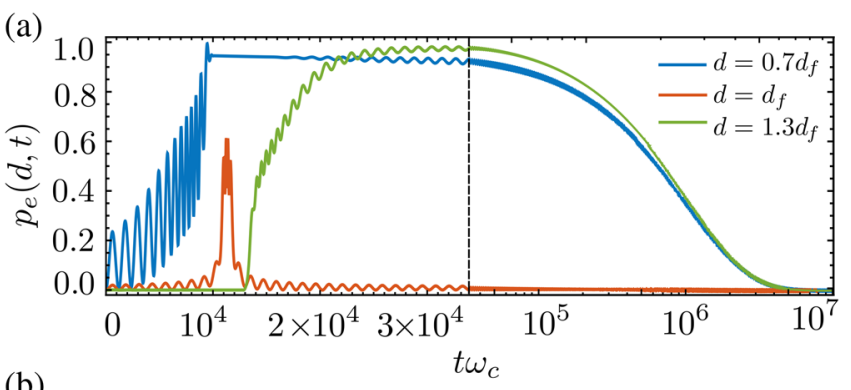

(b)

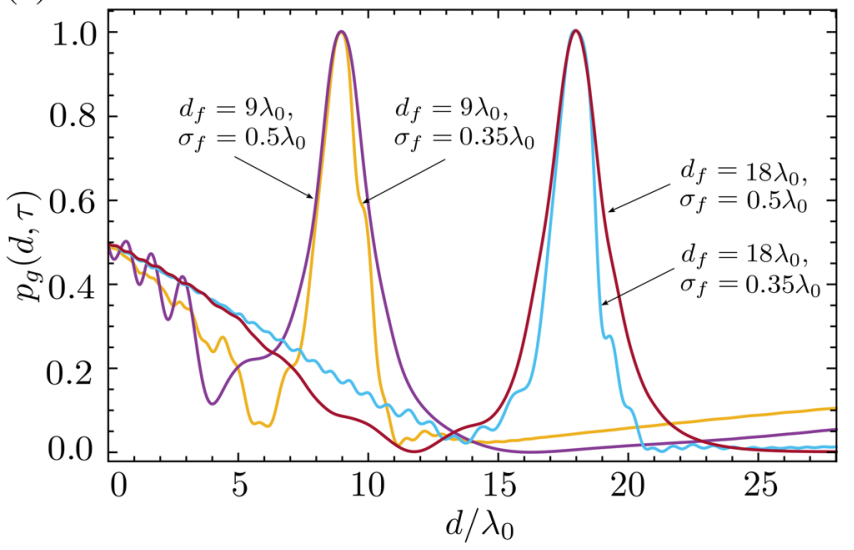

FIG. 2. (a) Excited state population of a qubit of frequency $\omega_{q}=\omega_{0}$ as a function of time for different positions of the qubit. We choose $\omega_{0} / \omega_{c}=1.005, \Gamma / \omega_{0}=10^{-6}, d_{f} / \lambda_{0}=18$, $\sigma_{f} / \lambda_{0}=0.35, \Omega_{0} / \omega_{c}=0.038$, and $\phi=0$. (b) Ground-state population of the qubit as a function of the on-axis distance $d$ from the origin, for different values of $d_{f}$ and $\sigma_{f}$ (see inset) and at a time $\tau(d)=2 t_{f}+\eta d / v$ (see main text). For (b) we fix $\omega_{0} / \omega_{c}=1.005$ and $\Omega_{0} / \omega_{c}=0.038\left(\Omega_{0} / \omega_{c}=0.030\right)$ for the curves with $\sigma_{f} / \lambda_{0}=0.35\left(\sigma_{f} / \lambda_{0}=0.5\right)$.

assuming usual spontaneous emission rates $\Gamma / \omega_{q} \lesssim 10^{-4}$. Figure 2(b) shows the ground state population of the qubit $p_{g}(d, t)=\operatorname{tr}[\hat{\rho}(t)|g\rangle\langle g|]$ as a function of the position $d$ of the qubit, at a time $\tau(d)$, such that $t_{f} \ll \tau(d) \ll \Gamma^{-1}$, that is, after the interaction with the pulse but before the decay of the qubit. As shown in the Supplemental Material [25], Fig. 2(b) does not depend on $\Gamma$ in the regime $\Gamma / \omega_{q} \lesssim 10^{-5}$. The plot shows different curves for different values of $d_{f}$ and $\sigma_{f}$. The ground-state population features a peak of height one centered at the compression distance of the pulse $d=d_{f}$ that is narrower the smaller the value of $\sigma_{f}$. The peak manifests that the self-compressing chirped pulse prepares a position-dependent state with a spatial resolution $\sigma_{q}$ (the width of the probability peak) that, as further discussed below, is given by $\sigma_{q} / \sigma_{f} \approx 1.34$ and thus can be smaller than $\lambda_{q} \equiv 2 \pi c / \omega_{q}$. Hence, the proposed selfcompressing chirped pulses can be used to perform remote subwavelength addressing of quantum emitters with a resolution length scale given by $\sigma_{q} \propto \sigma_{f}$.

The dynamics shown in Fig. 2 can be understood in the context of Landau-Zener (LZ) processes $[33,34]$. To this end, we consider the Hamiltonian (6) in the rotating wave approximation and write $\Omega(d, t) \equiv g(d, t) \exp [i \varphi(d, t)]$, 
where both functions $g(d, t)$ and $\varphi(d, t)$ are real and depend on the amplitude and phase of the electromagnetic pulse, respectively. One then moves to a rotating frame given by the unitary transformation $\hat{U}(t)=\exp \left[-i \varphi(d, t) \hat{\sigma}_{z} / 2\right]$, which converts the Hamiltonian (6) into

$$
\frac{\hat{H}_{\mathrm{LZ}}}{\hbar}=\left(\frac{\omega_{0}}{2}+\Delta(d, t)\right) \hat{\sigma}_{z}+\frac{g(d, t)}{2}\left(\hat{\sigma}_{+}+\hat{\sigma}_{-}\right),
$$

where $\Delta(d, t) \equiv \partial_{t} \varphi(d, t) / 2$ for $\omega_{q}=\omega_{0}$. In Eq. (7), the qubit detuning $\Delta(d, t)$ (Rabi coupling $g(d, t)$ ) is timedependent due to the chirping (time-dependent amplitude) of the electromagnetic pulse. The results shown in Fig. 2 can be explained in the dressed-state picture of Eq. (7). As further illustrated in [25], within the time interval at which the coupling $g>0$ and hence an energy gap opens between the dressed energies, the detuning $\Delta$ undergoes a single change (two changes) of sign whenever the qubit is out of focus $\left|d-d_{f}\right| \gg \sigma_{f}$ (on focus $\left|d-d_{f}\right| \ll \sigma_{f}$ ). In both regimes, the process is adiabatic. Consequently, the outof-focus qubit goes forth in the lower dressed-energy branch. Hence, after the pulse has passed and the energy gap closes $(g=0)$, the qubit ends up in the excited state. However, in the case when the qubit is on focus, it goes forth and back in the lower dressed-energy branch, thus ending in the ground state. For distances in the crossover regime $\left|d-d_{f}\right| \approx \sigma_{f}$ the process includes nonadiabatic transitions as the gap closes while the detuning changes sign. By comparing the timescales at which $\Delta$ changes sign and at which the gap opens due to the coupling $g$, we can estimate and numerically verify that the width of the peak in Fig. 2(b) is given by the above-quoted value of $\sigma_{q} / \sigma_{f} \approx 1.34$ [25].

Our results also hold for more complex quantum emitters, such as the nonlinear harmonic oscillator that models a transmon qubit [35]. The Hamiltonian describing the interaction of the quantum emitter with the chirped electromagnetic field pulse is now given by

$\frac{\hat{H}}{\hbar}=\omega_{\mathrm{q}} \hat{b}^{\dagger} \hat{b}+\frac{\alpha}{2}\left[\left(\hat{b}^{\dagger} \hat{b}\right)^{2}-\hat{b}^{\dagger} \hat{b}\right]+\frac{\Omega(d, t)}{2} \hat{b}^{\dagger}+\frac{\Omega^{*}(d, t)}{2} \hat{b}$,

where $\hat{b}\left(\hat{b}^{\dagger}\right)$ is a bosonic annihilation (creation) operator, $\alpha$ is the anharmonicity coefficient, and $\Omega(d, t)=$ $-2 d_{q} E(d, t) / \hbar$, where $d_{q}$ is the dipole moment of the anharmonic quantum emitter. One can then numerically solve the Born-Markov master equation $\partial_{t} \hat{\rho}=$ $(i \hbar)^{-1}[\hat{H}, \hat{\rho}]+\Gamma\left(\hat{b} \hat{\rho} \hat{b}^{\dagger}-\left[\hat{b}^{\dagger} \hat{b}, \hat{\rho}\right]_{+} / 2\right)$ in a truncated sufficiently large Hilbert space. We assume the initial state is $\hat{\rho}(0)=|0\rangle\langle 0|$, where $\hat{b}^{\dagger} \hat{b}|n\rangle=n|n\rangle$ with $n=0,1,2, \ldots$ In Fig. 3 we plot the excitation probability of the state $|n\rangle$, namely, $p_{n}(t, d) \equiv\langle n|\hat{\rho}(t)| n\rangle$, as a function of $d$. The population of the ground state features a peak around the compression position of the pulse, analogously to the

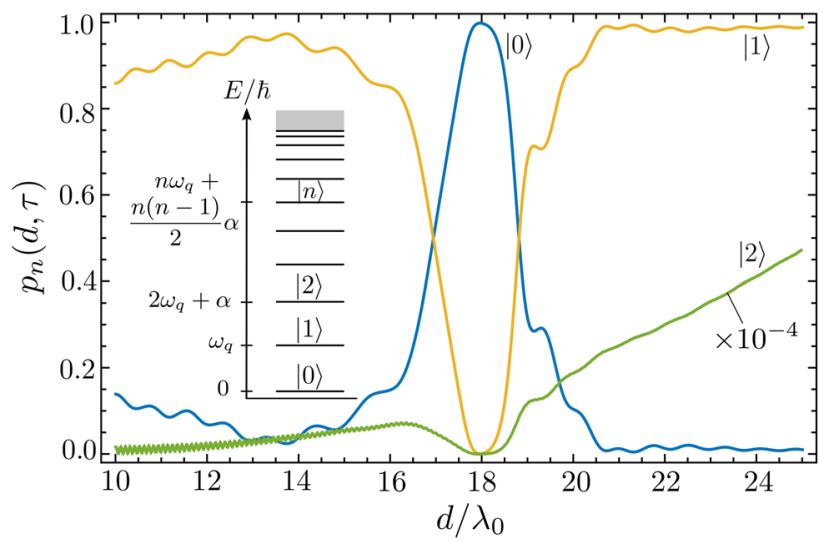

FIG. 3. Population of the internal states of an oscillator with anharmonicity $\alpha / \omega_{q}=-0.05$ interacting with the pulse as a function of the distance $d$ to the center of the waveguide, at a time $\tau(d)=2 t_{f}+\eta d / v$, such that $t_{f} \ll \tau(d) \ll \Gamma^{-1}$ with $\Gamma / \omega_{q}=10^{-6}$. Parameters used: $\omega_{q} / \omega_{c}=\omega_{0} / \omega_{c}=1.005$, $d_{f} / \lambda_{0}=18, \sigma_{f} / \lambda_{0}=0.35, \Omega_{0} / \omega_{c}=0.038, \phi=0$.

two-level quantum emitter. Note that the asymmetry of the electromagnetic pulse before and after the compression distance is imprinted in the excited states of the anharmonic quantum oscillator.

So far, we have analyzed the interaction of chirped electromagnetic pulses with a single quantum emitter as a function of its position in the waveguide. As shown in [25], our results hold in the case of an ensemble of many quantum emitters, as illustrated in Fig. 1(a), in the regime where the number of photons in the electromagnetic pulse is much larger than the number of quantum emitters and the single-photon coupling rate is weak. In this regime, both the interactions between quantum emitters and their backaction on the electromagnetic pulse, i.e., the total field scattered by the emitters can be neglected. According to our conservative estimation in [25], our results should hold for an ensemble of at least $\approx 10$ qubits for the parameters used in Figs. 2 and 3. The regime of few-photon pulses [36-38] or strongly coupled quantum emitters, which is notably challenging to approach theoretically due to interesting emerging quantum phenomena [39-42] is, in our opinion, a very interesting direction for further research.

Our results are relevant in many platforms where other options such as transverse driving are difficult (e.g., photonic crystals surrounded by a band-gapped medium), harmful (e.g., photodamage in hybrid metal-dielectric waveguides), incapable of individual addressing (e.g., closely packed qubit ensembles), or the source of unwanted decoherence (e.g., in superconducting circuits). The selfcompressing behavior described in this Letter only relies on free propagation in a quadratic dispersion relation and is thus not specific to the electromagnetic field. An interesting outlook of our work is to explore similar self-compressing dynamics in other systems with quadratic spectrum, collective quasiparticles such as bulk plasmons [43] or 
exciton polaritons [44,45], and even quantum technological platforms such as cavity arrays [46] and atoms in optical lattices [47]. By providing new probing and controlling capabilities at the quantum level, self-compressing pulses could thus become a relevant asset for quantum technologies in the future.

We acknowledge discussions with M. L. Juan, G. Kirchmair, A. Sharafiev, and M. Zanner. C. G.-B. acknowledges funding from the EU Horizon 2020 program under the Marie Skłodowska-Curie Grant Agreement No. 796725 (PWAQUTEC). J. J. G.-R. acknowledges support form Project No. PGC2018-094792-B-I00 (MCIU/AEI/ FEDER, UE), CAM/FEDER Project No. S2018/TCS4342 (QUITEMAD-CM), and the Quantum Technology Platform PTI-001 (CSIC).

*Corresponding author

silvia.casulleras-guardia@uibk.ac.at

[1] A. Albrecht, L. Henriet, A. Asenjo-Garcia, P. B. Dieterle, O. Painter, and D. E. Chang, New J. Phys. 21, 025003 (2019).

[2] N. M. Sundaresan, R. Lundgren, G. Zhu, A. V. Gorshkov, and A. A. Houck, Phys. Rev. X 9, 011021 (2019).

[3] V. S. Ferreira, J. Banker, A. Sipahigil, M. H. Matheny, A. J. Keller, E. Kim, M. Mirhosseini, and O. Painter, arXiv:2001.03240.

[4] A. Vrajitoarea, Z. Huang, P. Groszkowski, J. Koch, and A. A. Houck, Nat. Phys. 16, 211 (2020).

[5] I. Carusotto, A. A. Houck, A. J. Kollár, P. Roushan, D. I. Schuster, and J. Simon, Nat. Phys. 16, 268 (2020).

[6] Y. Schön, J. N. Voss, M. Wildermuth, A. Schneider, S. T. Skacel, M. P. Weides, J. H. Cole, H. Rotzinger, and A. V. Ustinov, npj Quantum Mater. 5, 18 (2020).

[7] P. Winkel, I. Takmakov, D. Rieger, L. Planat, W. HaschGuichard, L. Grünhaupt, N. Maleeva, F. Foroughi, F. Henriques, K. Borisov, J. Ferrero, A. V. Ustinov, W. Wernsdorfer, N. Roch, and I. M. Pop, Phys. Rev. Applied 13, 024015 (2020).

[8] E. Kim, X. Zhang, V. S. Ferreira, J. Banker, J. K. Iverson, A. Sipahigil, M. Bello, A. Gonzalez-Tudela, M. Mirhosseini, and O. Painter, Phys. Rev. X 11, 011015 (2021).

[9] P. Lodahl, A. F. van Driel, I. S. Nikolaev, A. Irman, K. Overgaag, D. Vanmaekelbergh, and W. L. Vos, Nature (London) 430, 654 (2004).

[10] T. Lund-Hansen, S. Stobbe, B. Julsgaard, H. Thyrrestrup, T. Sünner, M. Kamp, A. Forchel, and P. Lodahl, Phys. Rev. Lett. 101, 113903 (2008).

[11] P. Lodahl, S. Mahmoodian, and S. Stobbe, Rev. Mod. Phys. 87, 347 (2015).

[12] J. S. Douglas, H. Habibian, C. L. Hung, A. V. Gorshkov, H. J. Kimble, and D. E. Chang, Nat. Photonics 9, 326 (2015).

[13] A. González-Tudela, C. L. Hung, D. E. Chang, J. I. Cirac, and H. J. Kimble, Nat. Photonics 9, 320 (2015).

[14] A. González-Tudela, V. Paulisch, D. E. Chang, H. J. Kimble, and J. I. Cirac, Phys. Rev. Lett. 115, 163603 (2015).

[15] V. Paulisch, H. J. Kimble, and A. González-Tudela, New J. Phys. 18, 043041 (2016).
[16] J. D. Hood, A. Goban, A. Asenjo-Garcia, M. Lu, S.-P. Yu, D. E. Chang, and H. J. Kimble, Proc. Natl. Acad. Sci. U. S. A. 113, 10507 (2016).

[17] P. Lodahl, S. Mahmoodian, S. Stobbe, A. Rauschenbeutel, P. Schneeweiss, J. Volz, H. Pichler, and P. Zoller, Nature (London) 541, 473 (2017).

[18] E. Sánchez-Burillo, D. Zueco, L. Martín-Moreno, and J. J. García-Ripoll, Phys. Rev. A 96, 023831 (2017).

[19] M. Bello, G. Platero, J. I. Cirac, and A. González-Tudela, Sci. Adv. 5, eaaw0297 (2019).

[20] Y. Silberberg, Opt. Lett. 15, 1282 (1990).

[21] P. Chernev and V. Petrov, Opt. Lett. 17, 172 (1992).

[22] X. D. Cao, G. P. Agrawal, and C. J. McKinstrie, Phys. Rev. A 49, 4085 (1994).

[23] M.-A. Lemonde, S. Meesala, A. Sipahigil, M. J. A. Schuetz, M. D. Lukin, M. Loncar, and P. Rabl, Phys. Rev. Lett. 120, 213603 (2018).

[24] O. Romero-Isart, L. Clemente, C. Navau, A. Sanchez, and J. I. Cirac, Phys. Rev. Lett. 109, 147205 (2012).

[25] See Supplemental Material at http://link.aps.org/ supplemental/10.1103/PhysRevLett.126.103602 for an example of implementation of the pulse Eq. (2) in a hollow waveguide and a photonic crystal, a discussion about the extension to $N$ qubits, and additional figures, which includes Refs. [24,26-30].

[26] P. Maurer, J. Prat-Camps, J. I. Cirac, T. W. Hänsch, and O. Romero-Isart, Phys. Rev. Lett. 119, 043904 (2017).

[27] W. C. Chew, Waves and Fields in Inhomogenous Media (Wiley-IEEE Press, New York, 1995).

[28] C.-T. Tai, Dyadic Green Functions in Electromagnetic Theory, IEEE Series on Electromagnetic Waves (IEEE, New York, 1994).

[29] J. D. Joannopoulos, S. G. Johnson, J. N. Winn, and R. D. Meade, Photonic Crystals: Molding the Flow of Light. (Princeton University Press, Princeton, NJ, 2018).

[30] P. Hadley, Molecular and Solid State Physics. Light in a Layered Material (2018), http://lampx.tugraz.at/ hadley/ ss1/dbr/index.php [Accessed May 2020].

[31] M. Dalmonte, S. I. Mirzaei, P. R. Muppalla, D. Marcos, P. Zoller, and G. Kirchmair, Phys. Rev. B 92, 174507 (2015).

[32] D. Zoepfl, P. R. Muppalla, C. M. F. Schneider, S. Kasemann, S. Partel, and G. Kirchmair, AIP Adv. 7, 085118 (2017).

[33] C. Zener and R. H. Fowler, Proc. R. Soc. A 137, 696 (1932).

[34] N. V. Vitanov and B. M. Garraway, Phys. Rev. A 53, 4288 (1996).

[35] J. Koch, T. M. Yu, J. Gambetta, A. A. Houck, D. I. Schuster, J. Majer, A. Blais, M. H. Devoret, S. M. Girvin, and R. J. Schoelkopf, Phys. Rev. A 76, 042319 (2007).

[36] B. Q. Baragiola, R. L. Cook, A. M. Brańczyk, and J. Combes, Phys. Rev. A 86, 013811 (2012).

[37] A. H. Kiilerich and K. Mølmer, Phys. Rev. Lett. 123, 123604 (2019).

[38] A. H. Kiilerich and K. Mølmer, Phys. Rev. A 102, 023717 (2020).

[39] T. Shi, Y. Chang, and J. J. García-Ripoll, Phys. Rev. Lett. 120, 153602 (2018).

[40] T. Shi, D. E. Chang, and J. I. Cirac, Phys. Rev. A 92, 053834 (2015).

[41] T. Shi and C. P. Sun, Phys. Rev. B 79, 205111 (2009). 
[42] S. Mahmoodian, G. Calajó, D. E. Chang, K. Hammerer, and A. S. Sørensen, Phys. Rev. X 10, 031011 (2020).

[43] J. Quinn, Nucl. Instrum. Methods Phys. Res., Sec. B 96, 460 (1995).

[44] J. Kasprzak, M. Richard, S. Kundermann, A. Baas, P. Jeambrun, J. M. J. Keeling, F. M. Marchetti, M. H. Szymańska, R. André, J. L. Staehli, V. Savona, P. B. Littlewood, B. Deveaud, and L. S. Dang, Nature (London) 443, 409 (2006).
[45] M. Ramezani, A. Halpin, A. I. Fernández-Domínguez, J. Feist, S. R.-K. Rodriguez, F. J. Garcia-Vidal, and J. G. Rivas, Optica 4, 31 (2017).

[46] M. Hartmann, F. Brandão, and M. Plenio, Laser Photonics Rev. 2, 527 (2008).

[47] A. W. Glaetzle, K. Ender, D. S. Wild, S. Choi, H. Pichler, M. D. Lukin, and P. Zoller, Phys. Rev. X 7, 031049 (2017). 